\title{
RELAY-BASED AUTOTUNING WITH ALGEBRAIC CONTROL DESIGN
}

\author{
Roman Prokop, Jiř́i Korbel and Zdenka Prokopová \\ Faculty of Applied Informatics, \\ Tomas Bata University in Zlín, \\ Nad Stráněmi 4511, 76005 Zlín, Czech Republic \\ E-mail: prokop@fai.utb.cz
}

\section{KEYWORDS}

Autotuning, Algebraic control design, Pole-placement problem, PID controller, Relay.

\begin{abstract}
In this paper, a combination of relay feedback identification and an algebraic control design method for stable systems is studied. Models with up to three parameters are estimated by means of a single asymmetrical relay experiment. Then a stable low order transfer function is identified. Then the controller is analytically derived from general solutions of Diophantine equations in the ring of proper and stable rational functions. The controller parameters are tuned through a pole-placement problem as a desired multiple root of the characteristic closed loop equation. A first order identification yields a PI-like controllers while a second order identification generates PID ones. The approach enables a scalar tuning parameter $\mathrm{m}>0$ which can be adjusted by various principles.
\end{abstract}

\section{INTRODUCTION}

Since industrial processes are usually complex and nonlinear the task to control their loops properly becomes difficult and challenging. Moreover, the majority of controllers used in industrial applications have been still of the PID type. The practical advantages of PID controllers can be seen in a simple structure, in an understandable principle and in control capabilities. It is widely known that PID controllers are quite resistant to changes in the controlled process without meaningful deterioration of the loop behavior. A solution for qualified choice of controller parameters can be seen in automatic tuning of PID controllers.

The development of various autotuning principles was started by a simple symmetrical relay feedback experiment proposed by Åström and Hägglund, 1984. The ultimate gain and ultimate frequency are then used for adjusting of parameters by common known ZieglerNichols rules. During the period of more than two decades, many studies have been reported to extend and improve autotuners principles; see e.g. (Åströn and Hägglung, 1995; Ingimundarson and Hägglund, 2000; Majhi and Atherton, 1998; Morilla and Gonzáles, 2000). The extension in an experimental phase was performed in (Yu, 1999; Pecharromán and Pagola, 2000; Kaya and Atherton, 2001) by an asymmetry and hysteresis of a relay, see (Thyagarajan and $\mathrm{Yu}, 2002$; Kaya and Atherton, 2001), and experiments with asymmetrical and dead-zone relay feedback are reported in (Vítečková and Víteček, 2004; Vyhlídal, 2000). Also, various control design principles and rules can be investigated in mentioned references. Nowadays, almost all commercial industrial PID controllers provide the feature of autotuning.

This paper is focused on a novel combination for autotunig method of PI and PID controllers. The method combines an asymmetrical relay identification experiment and a control design method which is based on a pole-placement principle. The pole placement problem is formulated through a Diophantine equation and it is tuned by an equalization setting proposed in (Gorez and Klán, 2000).

\section{RELAY FEEDBACK ESTIMATION}

The estimation of the process or ultimate parameters is a crucial point in all autotuning principles. The relay feedback test can utilize various types of relay for the parameter estimation procedure. The classical relay feedback test (Åström and Hägglund, 1984) proposed for stable processes a symmetrical relay without hysteresis. Then the critical (ultimate) values can be estimated and a control design can follow.

Asymmetrical relays with or without hysteresis bring further progress (Yu, 1999; Kaya and Atherton, 2001). After the relay feedback test, the estimation of process parameters can be performed. A typical data response of such relay experiment is depicted in Figure1. The relay asymmetry is required for the process gain estimation (2) while a symmetrical relay would cause the zero division in the appropriate formula.

In this paper, an asymmetrical relay with hysteresis was used. This relay enables to estimate transfer function parameters as well as a time delay term. For the purpose of this contribution the time delay was not utilized.

The model for first order (stable) systems plus dead time (FOPDT) is supposed in the form: 


$$
G(s)=\frac{K}{T s+1} \cdot e^{-\Theta s}
$$

and the process gain can be computed by the relation (Vyhlídal, 2000):

$$
K=\frac{\int_{0}^{i T_{y}} y(t) d t}{\int_{0}^{i T_{y}} u(t) d t} ; \quad i=1,2,3, \ldots
$$

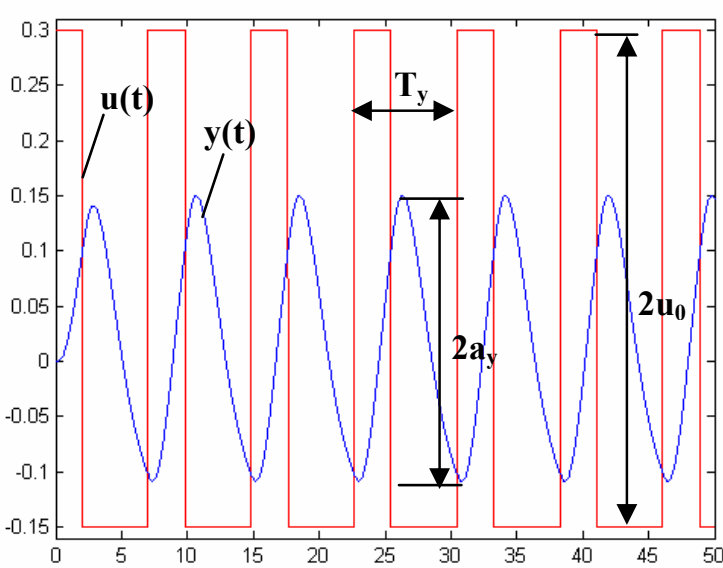

Figure 1: Asymmetrical relay oscillation of stable process

The time constant and time delay terms are given by (Vítečková and Víteček, 2004):

$$
\begin{aligned}
& T=\frac{T_{y}}{2 \pi} \cdot \sqrt{\frac{16 \cdot K^{2} \cdot u_{0}^{2}}{\pi^{2} \cdot a_{y}^{2}}-1} \\
& \Theta=\frac{T_{y}}{2 \pi} \cdot\left[\pi-\operatorname{arctg} \frac{2 \pi T}{T_{y}}-\operatorname{arctg} \frac{\varepsilon}{\sqrt{a_{y}^{2}-\varepsilon^{2}}}\right]
\end{aligned}
$$

where $\mathrm{a}_{\mathrm{y}}$ and $\mathrm{T}_{\mathrm{y}}$ are depicted in Fig. 1 and $\varepsilon$ is hysteresis.

Similarly, the second order model plus dead time (SOPDT) is assumed in the form:

$$
G(s)=\frac{K}{(T s+1)^{2}} \cdot e^{-\Theta s}
$$

The gain is given by (2), the time constant and time delay term can be estimated according to (Vítečková and Víteček, 2004) by the relation:

$$
\begin{aligned}
& T=\frac{T_{y}}{2 \pi} \cdot \sqrt{\frac{4 \cdot K \cdot u_{0}}{\pi \cdot a_{y}}-1} \\
& \Theta=\frac{T_{y}}{2 \pi} \cdot\left[\pi-2 \operatorname{arctg} \frac{2 \pi T}{T_{y}}-\operatorname{arctg} \frac{\varepsilon}{\sqrt{a_{y}^{2}-\varepsilon^{2}}}\right]
\end{aligned}
$$

\section{ALGEBRAIC CONTROLLER DESIGN}

The control design is based on the fractional approach; see e.g. (Vidyasagar, 1987; Kučera, 1993; Prokop and Corriou, 1997; Prokop, et al., 2002). Any transfer function $G(s)$ of a (continuous-time) linear system is expressed as a ratio of two elements of $R_{P S}$. The set $R_{P S}$ means the ring of (Hurwitz) stable and proper rational functions. Traditional transfer functions as a ratio of two polynomials can be easily transformed into the fractional form simply by dividing, both the polynomial denominator and numerator by the same stable polynomial of the order of the original denominator. Then all transfer functions can be expressed by the ratio:

$$
\begin{aligned}
& G(s)=\frac{b(s)}{a(s)}=\frac{\frac{b(s)}{(s+m)^{n}}}{\frac{a(s)}{(s+m)^{n}}}=\frac{B(s)}{A(s)} \\
& n=\max (\operatorname{deg}(a), \operatorname{deg}(b)), \quad m>0
\end{aligned}
$$

Then all feedback stabilizing controllers according to Figure 2 are given by a general solution of the Diophantine equation:

$$
A P+B Q=1
$$

which can be expressed with $\mathrm{Z}$ free in $\mathrm{R}_{\mathrm{PS}}$ :

$$
\frac{Q}{P}=\frac{Q_{0}-A Z}{P_{0}+B Z}
$$

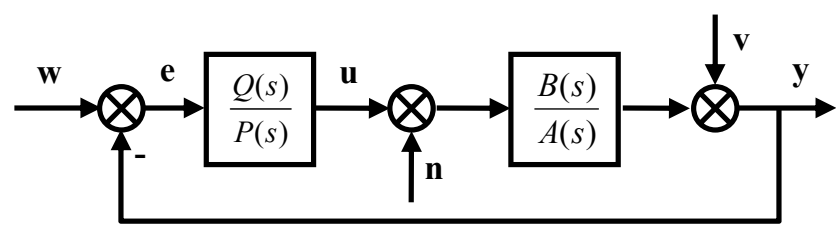

Figure 2: Feedback (1DOF) control loop

Asymptotic tracking is then ensured by the divisibility of the denominator $P$ in (9) by the denominator of the reference $w=\frac{G}{F}$ which is supposed in the form:

$$
F_{w}=\frac{s}{s+m} ; \quad m>0
$$

The set of reference signals with denominator (10) represents all stepwise signals which are most frequent references. The divisibility is achieved by a suitable choice of rational function $\mathrm{Z}$ in (9), see (Prokop, et al., 2002). 


\section{PI AND PID-LIKE CONTROLLERS}

The control design for first order systems (1) gives the Diophantine equation (8) in the form:

$$
(T s+1) p_{0}+K q_{0}=s+m
$$

and the general solution is given by:

$$
\begin{aligned}
& P=\frac{1}{T}+\frac{K}{s+m} \cdot Z \\
& Q=\frac{T m-1}{T K}-\frac{T s+1}{s+m} \cdot Z
\end{aligned}
$$

where $\mathrm{Z}$ is free in the ring $\mathrm{R}_{\mathrm{PS}}$. Asymptotic tracking is achieved by the choice:

$$
Z=-\frac{m}{T K}
$$

and the resulting PI controller is in the form:

$$
C(s)=\frac{Q}{P}=\frac{q_{1} s+q_{0}}{s}
$$

where parameters $\mathrm{q}_{1}$ a $\mathrm{q}_{0}$ are given by:

$$
q_{1}=\frac{2 T m-1}{K} \quad q_{0}=\frac{T m^{2}}{K}
$$

For the SOPDT the design equation takes the form:

$$
(T s+1)^{2} \cdot s \cdot p_{0}+K \cdot\left(q_{2} s^{2}+q_{1} s+q_{0}\right)=(s+m)^{3}
$$

and after similar manipulations the resulting PID controller gives the transfer function:

$$
C(s)=\frac{Q}{P}=\frac{q_{2} s^{2}+q_{1} s+q_{0}}{s\left(s+p_{0}\right)}
$$

with parameters:

$$
\begin{aligned}
& p_{0}=\frac{1}{T^{2}} ; \quad q_{2}=\frac{3 T m-2}{K T} \\
& q_{1}=\frac{3 T^{2} m^{2}-1}{K T^{2}} ; \quad q_{0}=\frac{m^{3}}{K}
\end{aligned}
$$

For both systems FOPDT and SOPDT the scalar parameter $m>0$ seems to be a suitable ,tuning knob" influencing control behavior as well as robustness properties of the closed loop system.
The control law for the equalization method (Gorez and Klán, 2000) is supposed in the form of the PI controller:

$$
u(t)=K_{P} \cdot\left(e(t)+\frac{1}{T_{I}} \cdot \int e(\tau) d \tau\right)
$$

The PI controller parameters can be tuned by simple relations:

$$
K_{P}=\frac{1}{2 K} \quad T_{I}=0.4 \cdot T_{u}
$$

where $\mathrm{K}$ is process gain and $\mathrm{T}_{\mathrm{u}}$ is ultimate period.

The parameter $\mathrm{q}_{1}$ in (15) represents the controller gain and is tuned by the "equalization" principle. The choice for the tuning parameter $m>0$ in the case of PI controller is given by:

$$
m=\frac{3}{4 \cdot T}
$$

Naturally, this choice of $m$ does not yield the same value of $T_{I}$ given by formula (20). However, the difference is not significant and the authors prefer the influence of the controller gain.

\section{EXAMPLES AND ANALYSIS}

The following three examples illustrate the situation where the controlled system is always estimated by the first order one (1) with or without time delay terms and the controller has a PI structure (14).

The first order system governed by the transfer function $\mathrm{G}(\mathrm{s})$ was after the relay experiment estimated by $\widetilde{G}(s)$ in the form:

$$
G(s)=\frac{2}{3 s+1} \quad \widetilde{G}(s)=\frac{2}{2.93 s+1}
$$

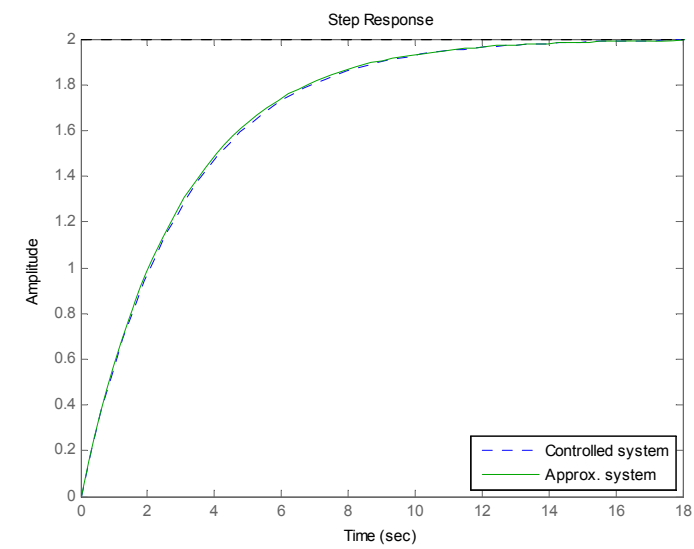

Figure 3: Step responses of nominal and estimated systems 
The PI controller was then generated for $\mathrm{m}=0,256$ by relations (14), (15) and the resulting control behavior are depicted in Figure 4.

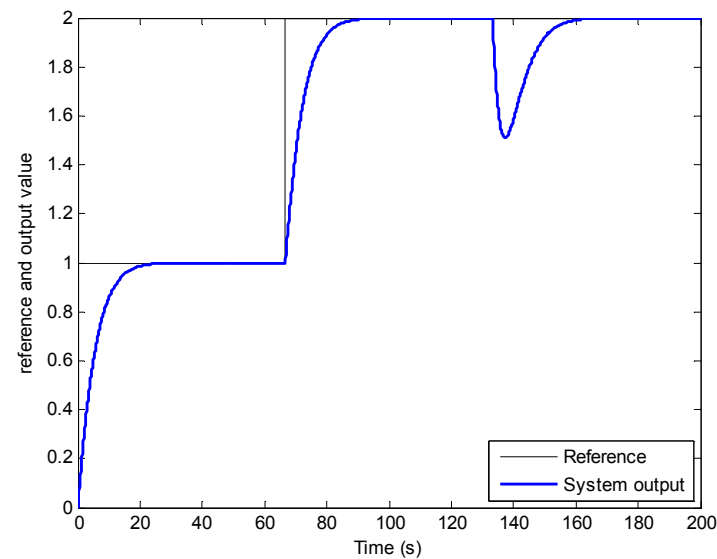

Figure 4: Control response for $\mathrm{m}=0.256$

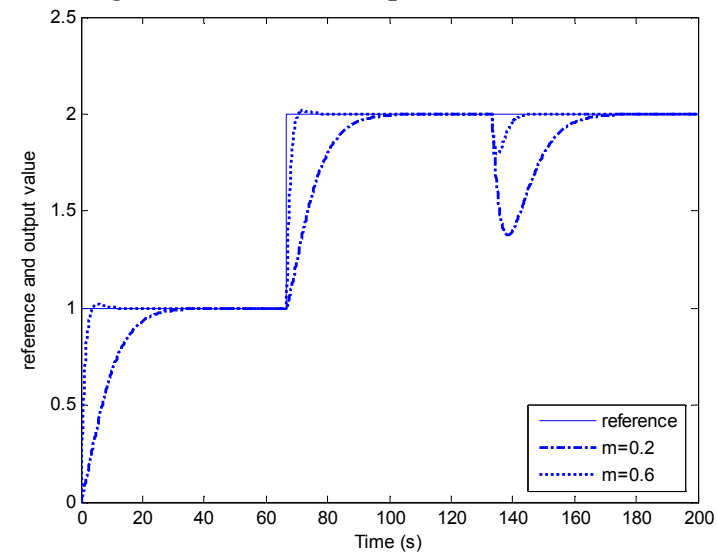

Figure 5: Control responses for two different values $\mathrm{m}>0$

The choice of the parameter $m>0$ can easily influence the control response, e.g. the increasing value of $m>0$ accelerate the control response but escalate overshoots. The situation for two values of $m>0$ is shown in Figure 5.

The next example represents the situation where a second order (stable) system G(s) was estimated by a first order model in the above mentioned relay experiment. Both transfer functions have the form:

$$
G(s)=\frac{3}{(2 s+1)^{2}} \quad \widetilde{G}(s)=\frac{3.02}{3.89 s+1} \cdot e^{-1.15 s}
$$

The PI controller generated from the approximated system $\widetilde{G}(s)$ for the scalar tuning parameter $\mathrm{m}=0.19$ and applied to the system $\mathrm{G}(\mathrm{s})$ gives the control response depicted in Figure 7.

Comparison of two control responses for two different values of the tuning parameter $\mathrm{m}$ can be seen in Figure 8 .

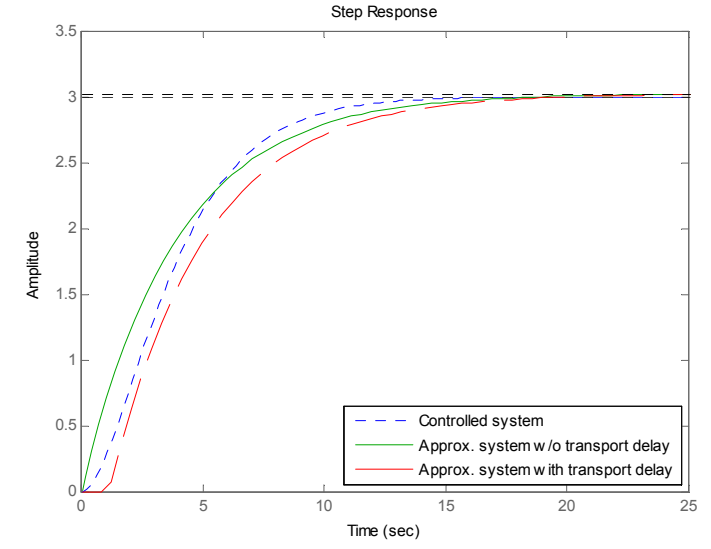

Figure 6: Step responses of systems (23)

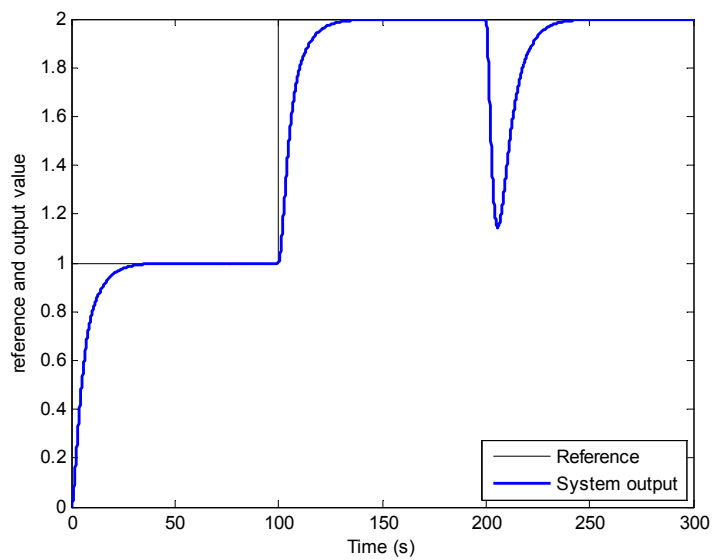

Figure 7: Control response for $\mathrm{m}=0.19$ for second order system $\mathrm{G}$ in (23)

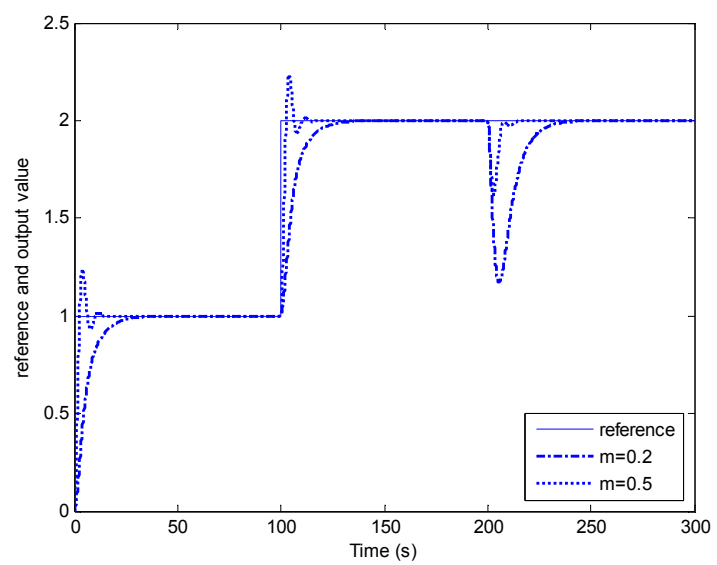

Figure 8: Control responses of the second order systems for two values $m>0$

As a third example a high order system with transfer function G(s) was supposed. Again, after the relay experiment, a first order estimation $\widetilde{G}(s)$ was identified, both governed by:

$$
G(s)=\frac{3}{(s+1)^{8}} \quad \tilde{G}(s)=\frac{3}{3.52 s+1} \cdot e^{-5.2 s}
$$


Naturally, the step response of the estimated system is different from the nominal system as depicted in Figure 9 because the time-delay is neglected. However, the control design for $\mathrm{m}=0.2$ gives the PI controller and the resulting control behavior is shown in Figure 10.

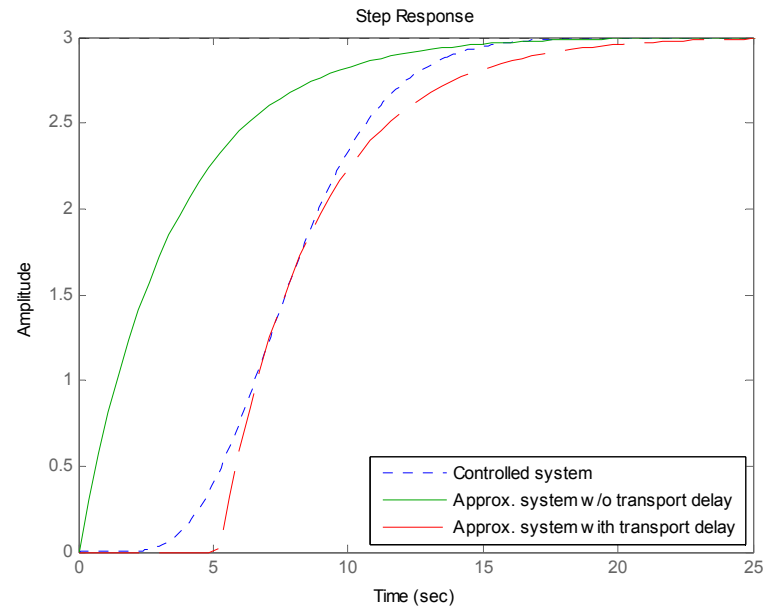

Figure 9: Step responses of systems (24)

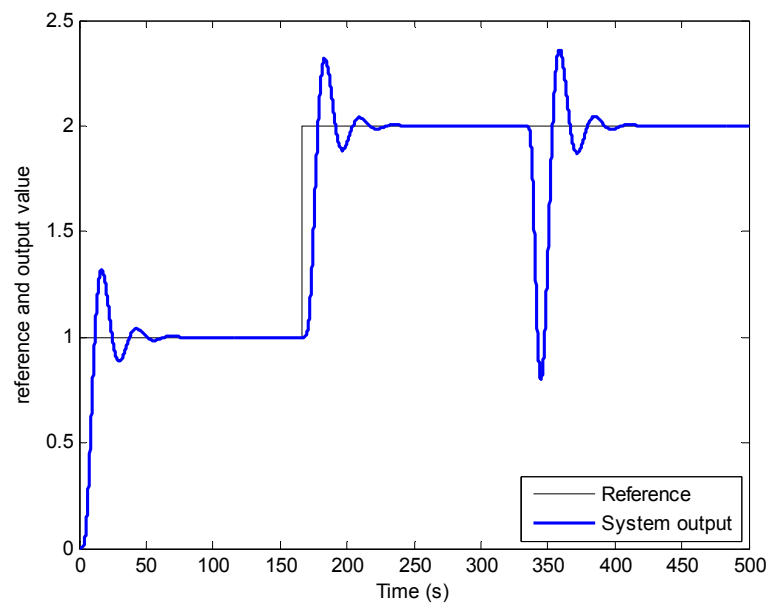

Figure 10: Control response for $\mathrm{m}=0.2$

Again, the tuning parameter $m>0$ can influence the control response. Figure 11 illustrates further control behavior for different PI controllers. In all simulations, the reference value is changed in $1 / 3$ of the simulation horizon and the load disturbance is injected in the $2 / 3$ of the simulation horizon.

As a last example, the fourth order controlled system is supposed in the form

$$
G(s)=\frac{2}{(0.5 s+1)^{4}}
$$

The second order identification relations (5) give the estimated transfer function:

$$
\tilde{\tilde{G}}(s)=\frac{2.007}{(0.612 s+1)^{2}} \cdot e^{-0.58 s}
$$

and Figure 12 shows the original and approximated step responses of (26) with and without the time delay term.

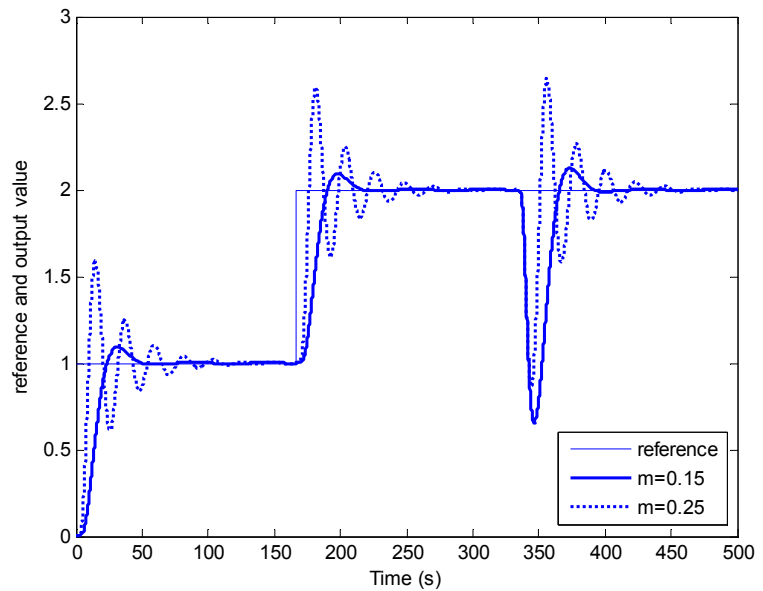

Figure 11: Control responses for two different values of $\mathrm{m}>0$

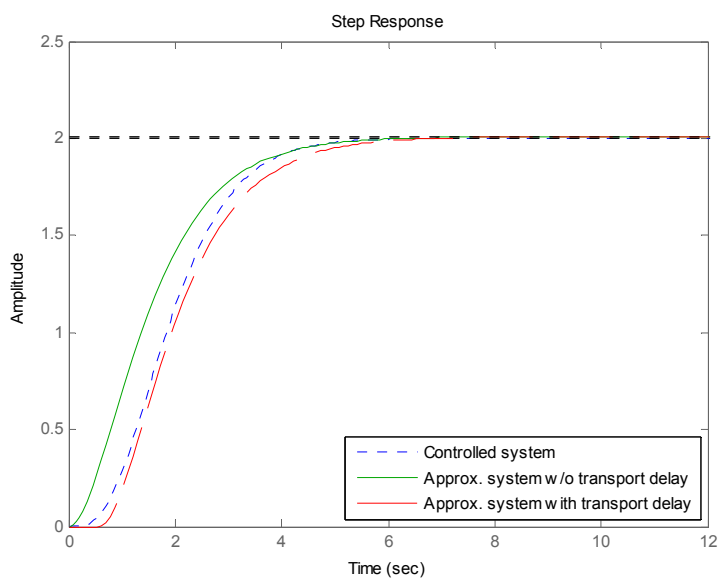

Figure 12: Step responses of systems (25) - (26)

The controller was designed for the estimated transfer function (26) according to SOPDT synthesis (16) (18). The final control responses for two different tuning parameters $m>0$ are depicted in Figure 13. Naturally, the controlled system in simulations was the original one in (25). Again, the influence of the scalar tuning parameter is obvious and apparent.

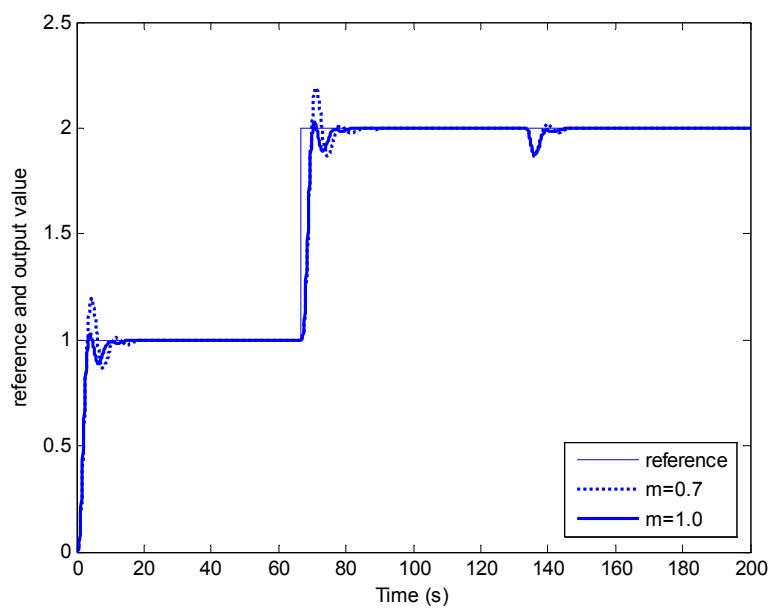

Figure 13: Control responses for SOPDT 


\section{CONCLUSION}

This contribution gives a new combination of relay feedback identification and a control design method as an autotuning principle. The estimation of a low order transfer function parameters is performed from asymmetric limit cycle data. The control synthesis is carried out through the solution of a linear Diophantine equation; see (Vidyasagar, 1987; Kučera, 1993; Prokop and Corriou, 1997; Prokop, et al., 2002). This approach brings a scalar tuning parameter which can be adjusted by various strategies. A first order estimated model generates PI-like controllers while a second order model gives a class of PID ones. The methodology is illustrated by several examples of various orders and dynamics.

\section{Acknowledgments}

This work was supported by the grant of Ministry of Education, Youth and Sports of the Czech Republic, MSM 7088352102.

\section{REFERENCES}

Åström, K.J. and T. Hägglund (1995). PID Controllers: Theory, Design and Tuning. Research Triangle Park, NC: Instrumental Society of America.

Garcia, R.F. and F.J.P. Castelo (2000). "A complement to autotuning methods on PID controllers," in Preprints of IFAC Workshop PID'00, pp. 101-104.

Gorez, R. and P. Klán (2000). "Nonmodel-based explicit design relations for PID controllers," in: Preprints of IFAC Workshop PID'00, pp. 141-146.

Hang, C.C., K.J. Åström and Q.C. Wang (2002). "Relay feedback auto-tuning of process controllers - a tutorial review," Journal of Process Control 12.

Ingimundarson, A. and T. Hägglund (2000). "Robust automatic tuning of an industrial PI controller for deadtime systems" In: Preprints of IFAC Workshop PID'00, 149-154.

Kaya, I. and D.P. Atherton (2001). "Parameter estimation from relay autotuning with asymmetric limit cycle data," in: Journal of Process Control, pp. 429-439.

Kučera, V. (1993). "Diophantine equations in control - A survey," Automatica, Vol. 29, pp. 1361-75.

Majhi, S. and D.P. Atherton (1998). "Autotuning and controller design for unstable time delay processes" In: Preprints of UKACC Int. Conf. on Control, 769-774.

Majhi, S. (2007). "Relay based identification of processes with time delay," Journal of Process Control 17.

Morilla, F., A. Gonzáles and N. Duro (2000). "Auto-tuning PID controllers in terms of relative damping" In: Preprints of IFAC Workshop PID'00, 161-166.

Panda, R.C., and Ch.-Ch. Yu (2003). "Analytical expressions for relay feedback responses," Journal of Process Control 13.

Pecharromán, R.R. and F.L. Pagola (2000). "Control design for PID controllers auto-tuning based on improved identification," in: Preprints of IFAC Workshop PID'00, pp. 89-94

Prokop, R. and J.P. Corriou (1997). "Design and analysis of simple robust controllers," Int. J. Control, Vol. 66, pp. 905-921.
Prokop, R., P. Husták, and Z. Prokopová (2002). "Simple robust controllers: Design, tuning and analysis," in: Preprints of 15th IFAC World Congress.

Thyagarajan, T. and Ch.Ch. Yu (2002). "Improved autotuning using shape factor from relay feedback," in: Preprints of IFAC World Congres.

Vidyasagar, M. (1987). Control system synthesis: a factorization approach. MIT Press, Cambridge, M.A.

Vítečková, M. and A. Víteček (2004), "Experimentální identifikace metodou relé,“ in: Automatizácia a informatizácia.

Vyhlídal, T. (2000). "Anisochronic first order model and its application to internal model control," in: ASR '2000 Seminar.

Yu, Ch.Ch. (1999). Autotuning of PID Controllers. Springer, London.

\section{AUTHOR BIOGRAPHIES}

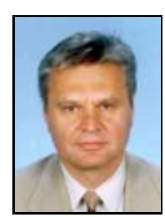

ROMAN PROKOP was born in Hodonin, Czech Republic in 1952. He graduated in Cybernetics from the Czech Technical University in Prague in 1976. He received post graduate diploma in 1983 from the Slovak Technical University. Since 1995 he has been at Tomas Bata University in Zlín, where he presently holds the position of full professor of the Department of Automation and Control Engineering and a vice-rector of the university. His research activities include algebraic methods in control theory, robust and adaptive control, autotuning and optimization techniques. His e-mail address is: prokop@fai.utb.cz.

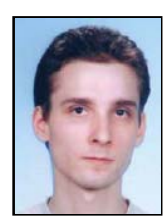

JIŘÍ KORBEL was born in Zlín, Czech Republic. He studied automatic control and informatics at the Tomas Bata University and graduated in 2004, now he is an post-graduate student and assistant at the Faculty of Applied Informatics in Zlín. His research activities include autotuning principles, algebraic and polynomial syntheses and modeling and simulations. His e-mail address is: korbel@fai.utb.cz.

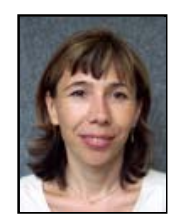

ZDENKA PROKOPOVÁ was born in Rimavská Sobota, Slovak Republic. She graduated from Slovak Technical University in 1988, with a master's degree in automatic control theory. Doctor's degree she has received in technical cybernetics in 1993 from the same university. Since 1995 she is working in Tomas Bata University in Zlin, Faculty of Applied Informatics. She is now working there as an associating professor. Research activities: mathematical modeling, simulation, control of technological systems, programming and application of database systems. Her e-mail address is: prokopova@fai.utb.cz. 